\title{
THE ROLE OF ALGEBRA IN TOPOLOGY $\dagger$
}

\section{BY SOLOMON LEFSCHETZ}

1. Introduction. The assertion is of ten made of late that all mathematics is composed of algebra and topology. It is not so widely realized that the two subjects interpenetrate so that we have an algebraic topology as well as a topological algebra.

The increasing importance of algebra in topology, a domain whose roots lie in a very different soil, signifies that here also the age of consolidation and coordination is upon us. My present object is primarily to show that a reasonable blend of the algebraic and topological points of view is possible. For this purpose I shall formalize my earlier procedure of immersion in variable euclidean spaces by means of which I introduced dual cycles into topology. We shall see that around this mode of attack it is possible to group many of the recent very interesting results of combinatorial topology.

\section{Algebra of Complexes}

2. Abstract Complexes. Abstract complexes have been investigated by various authors, notably in recent years by $\mathrm{J}$. W. Alexander, W. Mayer, and A. W. Tucker. $\ddagger$ While I shall lean particularly on Tucker's work, my discussion bears largely on simplicial complexes and their duals, the basic types in topology.

According to Tucker, then, an abstract complex $K$ is a set of elements $E$, its cells, partially ordered relative to a transitive geometric relation of incidence $\prec$ ("on the boundary of"), and with certain additional (algebraic) relations of incidence to be described presently. Each $E$ has a dimension $p$ which is a positive or negative integer and shall be frequently denoted by an index, as $E_{p}$. Moreover $E<E^{\prime}$ implies $\operatorname{dim} E<\operatorname{dim} E^{\prime}$. A p-chain of $K$ is a linear form

$$
C_{p}=x_{i} E_{p}^{i}
$$

$\dagger$ An address delivered at Duke University, December 30,1936, as the retiring presidential address, before the American Mathematical Society.

$\ddagger$ J. W. Alexander, Transactions of this Society, vol. 28 (1926), pp. 301-329; W. Mayer, Monatshefte für Mathematik und Physik, vol. 36 (1929), pp. 1-42, 219-258; A. W. Tucker, Annals of Mathematics, vol. 34 (1933), pp. 191-243. 
where $x_{i}$ is a member of a given additive abelian group (5). There is attached to $K$ a linear chain-function $F$, called a boundary operator and determined by

$$
F\left(E_{p}^{i}\right)=\left[E_{p}^{i}: E_{p-1}^{j}\right] E_{p-1}^{j}, \quad F\left(x_{i} E_{p}^{i}\right)=x_{i} F\left(E_{p}^{i}\right),
$$

where the incidence-number [ ] is an integer, which is $\neq 0$ only when $E_{p} \underline{i}_{1} \prec E_{p}^{i}$. The operator $F$ is subjected to the condition

$$
F F=0 .
$$

A chain $\Gamma_{p}$ such that $F\left(\Gamma_{p}\right)=0$, is a $p$-cycle. Owing to (3), any $F\left(C_{p+1}\right)$ is a $\Gamma_{p}$. The difference (factor) group $G_{p}=\left\{\Gamma_{p}\right\}$ $-\left\{F\left(C_{p+1}\right)\right\}$ is the pth homology group of $K$ over (s).

If we replace everywhere $E_{p}^{i}$ by $-E_{p}^{i}$, the relations just written remain true provided that all the incidence-numbers [ ] involving $E_{p}^{i}$ are changed in sign. We agree that under these conditions $K$ has not been changed, its cell $E_{p}^{i}$ having merely been reoriented.

Dualization. Consider a new aggregate $K^{*}=\left\{E_{i}^{p}\right\}$, where $E_{i}^{p} \longleftrightarrow E_{p}^{i}$ is a (1-1) correspondence. We assume: (a) $\operatorname{dim} E_{i}^{p}$ $=-\operatorname{dim} E_{p}^{i}=-p$; (b) $E_{i}^{p}<E_{j}^{q}$ when and only when $E_{q}^{j}<E_{p}^{i}$; (c) $\left[E_{j}^{p-1}: E_{i}^{p}\right]=(-1)^{p}\left[E_{p}^{i}: E_{p^{j}-1}\right]$. It is a simple matter to verify that the resulting operator $F$ for $K^{*}$ satisfies (3); hence $K^{*}$ is also a complex, the dual of $K$.

The changes in sign indicated under (c), which are convenient for some purposes, may be eliminated by reorienting the cells $E_{p}^{i}, p$ even. Therefore the relation between $K$ and $K^{*}$ is in fact symmetrical, and in particular $K^{* *}=K$. This symmetry lies at the root of all the duality properties in topology. $\dagger$

There subsist between the homology groups $G_{p}$ of $K$ and $G^{p}$ (for the dimension $-p$ ) of $K^{*}$ the duality relations of the Poincare or Pontrjagin types according to the choices of coefficient-groups. Thus if the coefficients are: integers for $K$, real numbers mod 1 for $K^{*}$, the groups $G_{p}$ and $G^{p}$ are one another's character-groups.

† Some recent authors (Alexander, Kolmogoroff, Whitney) bring in this dualism by referring the properties of $K^{*}$ back to $K$, through the transformation $E_{i}^{p} \rightarrow E_{p}^{i}$. As a consequence the operator $F$ of $K^{*}$ gives rise to a dual boundary operator $F^{*}$ for $K$, with $\operatorname{dim} F^{*}(C)=\operatorname{dim} C+1$. The choice of procedure is largely a matter of taste. 
3. Simplicial Complexes. The elements of a simplicial complex are the simplexes. An (abstract) $p$-simplex $\sigma_{p}$ is any finite collection of $p+1$ elements or vertices $a_{0}, \cdots, a_{p}$; its faces are the proper subcollections of the set. $\sigma$ is oriented by assigning an order to its vertices modulo an even permutation. A closed (abstract) simplicial complex is a collection $K=\{\sigma\}$ such that $\sigma \in K$ implies that every face of $\sigma \in K$. The incidence-numbers are determined by $[a \sigma: \sigma]=1$, under which (3) is verified.

A very suggestive description of $K$ and its dual may be given in terms of a certain algebra $\mathfrak{A}$. To define it we introduce new symbols $a_{i}^{-1, l}, a_{i}^{-1, r},+1,-1,0$ and shall assume that all the symbols $a$ other than $0, \pm 1$ obey the following rules of combination:

$$
\begin{gathered}
a a^{\prime}=-a^{\prime} a, \quad a^{2}=0, \quad a^{-1, l} a=1 ; \\
a^{-1, r}=-a^{-1, l} \quad\left(\text { hence } a a^{-1, r}=1\right) ;
\end{gathered}
$$

if neither a product $a \cdots a^{\prime}$ nor its inverse is made up of the vertices of a $\sigma \in K$, it is to be set equal to zero.

The symbols $a^{-1, l}, a^{-1, r}$ play the role of left and right inverses. Usually only the former shall be considered and I shall merely write $a^{-1}$ for $a^{-1, l}$. The only monomials that may occur are then of one of the types, $\pm \sigma= \pm a \cdots b, \pm \sigma^{-1}= \pm b^{-1} \cdots a^{-1}$. The products of any two of the monomials are obtained in the customary formal way and always give rise to monomials of one of the two types or to $\pm 1,0$. The associative law holds between products of $\sigma^{\text {'s }}$ or of $\sigma^{-1}$ 's alone, but not between products involving both types. Thus $\left(a^{-1, l} a\right) a^{-1, r}=a^{-1, r} \neq a^{-1, l}\left(a a^{-1, r}\right)$ $=a^{-1, l}$. It follows in particular from these rules of combination that $\left(c^{-1} \cdots b^{-1} a^{-1}\right)(a b \cdots c)=1$. For this reason we designate the first factor by $(a b \cdots c)^{-1}$. This is again a left-handed inverse, the exponent -1 taking here also the place of $-1, l$.

Each oriented $\sigma$ of $K$ determines a unique monomial product of positive powers which we shall likewise denote by $\sigma$. The $p$-chains over a group of coefficients (5) are the forms of degree $p$ with coefficients in $\$$. They are members of a ring $\Re$ (noncommutative and non-associative) whose elements are the polynomials in the base elements of $\mathfrak{A}$, with coefficients in $\mathbb{B}$.

In terms of the inverse symbols, we have

$$
F\left(\sigma_{p}\right)=\sum a_{i}^{-1} \cdot \sigma_{p} \quad \text { for } \quad p>0 ; \quad F\left(a_{i}\right)=0 .
$$


Hence

$$
F\left(C_{p}\right)=\sum a_{i}^{-1} \cdot C_{p} \text { for } p>0 ; \quad F\left(C_{0}\right)=0 .
$$

The direct verification of $(3)$ is immediate.

Notice that if $L$ is a closed subcomplex of $K$, and we consider only cells of $K-L$, setting all others equal to zero, the conditions for a complex are still verified. $K-I$ is a so-called open complex.

It is quite natural at this juncture to introduce the product $\sigma_{p}^{-1}$ as an abstract $(-p)$-cell, the inverse of $\sigma_{p}$, and adopt the following rules: (a) $\sigma_{p}^{-1} \prec \sigma_{q}^{-1}$ when and only when $\sigma_{q} \prec \sigma_{p}$; (b) the boundary operator is as before

$$
l\left(\sigma_{p}^{-1}\right)=\left(\sum a_{i}^{-1}\right) \sigma_{p}^{-1} \text { for every } p .
$$

The verification of (3) is again immediate; hence $K^{1}= \begin{cases}\sigma^{1} \\ \sigma^{\prime}\end{cases}$ is a complex. The incidence relations and numbers show that it is isomorphic with $K^{*}$. In the future we may therefore replace the dual elements by inverses and $K^{*}$ by $K^{\cdots-1} \cdot$

Augmented Complex. In certain applications it may be more convenient to define $\operatorname{dim} \sigma$ as equal to the number of vertices of $\sigma$. This may be done formally by considering 1 as a vertex of every $\sigma$, that is, by considering $\sigma$ as being in effect $1 \sigma$. The boundary relations are then chosen as

$$
F(C)=\sum a_{i}^{-1} \cdot C ; \quad F(x)=0, \quad x \in(\$)
$$

for every $C$. This is tantamount to the old rule wherein it took two points to make a zero-cycle, one being insufficient, whereas under our initial scheme every $F(a)=0$. Under these assumptions we shall call $K$ an augmented complex (see Tucker, loc. cit., p. 11).

Remark. Let $\Re$ be a Hausdorff space and $\left(a_{0}, \cdots, a_{r}\right)$ a finite covering of $\Re$ by open sets. The intersection diagram of the sets in the sense of Alexandroff is a simplicial complex $K$ with the $a$ 's as vertices, the rule being that $a_{i_{0}} \cdots a_{i_{p}} \in K$ whenever the sets indicated have an intersection $\neq 0$. The incidences in $K$ correspond to the inclusions $\supset$. Iet us now designate by $a_{i}{ }^{-1}$ the

$\dagger$ Quotients of the positive symbols have been utilized by M. H. A. Newman (Cambridge Philosophical Transactions, vol. 27 (1931), pp. 491.504), in the treatment of combinatorial intersections. 
closed set $\Re-a_{i}$. The aggregate $\left\{a_{i}^{-1}\right\}$ is merely any aggregate of non-intersecting closed sets. To $\prod a_{i} \neq 0$ there corresponds now $\prod\left(\Re-a_{i}^{-1}\right) \neq 0$ or $\sum a_{i}^{-1} \neq \Re$. It follows that the elements of the dual $K^{-1}$ represent the mutual inclusions of the sums with $\prec$ corresponding this time to $c$. This gives a noteworthy geometrical interpretation for the duals of simplicial complexes. It may be pursued still further, in the construction of the projectionspectra, and leads to a natural interpretation for the opposite types of homomorphisms (inverse and direct) associated with the ordinary and the dual cycles of $\Re$.

\section{SPHERES}

4. Duality. The duality properties of complexes and sets (duality theorem of Alexander and its extensions) are intimately connected with the topological properties of the complementary regions of their topological images in euclidean spaces or spheres. We may therefore expect to find a good approach to these questions in a "combinatorial" immersion of a given abstract complex into abstract spheres. This requires that we begin with a consideration of the latter.

The simplest abstract $n$-sphere $H_{n}$ is the sum of the proper faces of a $\sigma_{n+1}=a_{0} \cdots a_{n+1}$. We shall designate its dual by $H_{n}^{-1}$.

The complex $H_{n}$ is the most elementary combinatorial $n$ manifold $M_{n}$. Owing to its simplicity we may take full advantage of the known theory of manifolds, $\dagger$ which is moreover easily developed for this special case. As in the general theory, the first step is to take the barycentric subdivision $H_{n}^{\prime}$ of $H_{n}$, and to relate duality to $H_{n}^{\prime}$. The complex $H_{n}^{\prime}$ will have a single new vertex associated with each $a_{i_{0}} \cdots a_{i_{p}}$, and denoted by $\left(i_{0} \cdots i_{p}\right)$.

We verify that the simplexes $\sigma_{n-p}^{0}=\sigma_{p}^{-1} \sigma_{n+1}$ have the mutual incidences of the duals $\sigma_{p}^{*}$. It follows that the aggregate $\left\{\sigma_{p}^{-1} \sigma_{n+1}\right\}=H_{n}^{0}$ is a complex isomorphic with the dual of $H_{n}$. Notice that $\sigma_{n-p}^{0}$ is uniquely determined by the condition $\sigma_{p} \sigma_{n-p}^{0}=(-1)^{p+1} \sigma_{n+1}$.

On the other hand if we consider for $\sigma_{p}=a_{i_{0}} \cdots a_{i_{p}}$ the sum $\sigma_{n-p}^{1}$ of the $(n-p)$-simplexes of $H_{n}^{\prime}$ with vertices $\left(i_{0} \cdots i_{p}\right.$ $j \cdots k)$, we find that it is isomorphic with a barycentric sub-

$\dagger$ See Chapter III of my Colloquium Lectures, Topology, New York, 1930 (hereafter referred to merely as Topology). 
division of type $\sigma_{n-p}^{0}$. Therefore $\sigma_{n-p}^{1}$ is essentially an $(n-p)$ simplex. $\dagger$ We shall agree to orient its component $(n-p)$-simplexes (of $H_{n}^{\prime}$ ) concordantly with the subdivision of $\sigma_{n-p}^{0}$, and thus oriented we denote it by $\left(\sigma_{p}\right)^{*}$ or $\sigma_{p}^{*}$. The aggregate $\left\{\sigma^{*}\right\}$ is another isomorph of the dual of $H_{n}$, and it is this new complex which shall be designated temporarily by $H_{n}^{*}$.

Geometric Realization. The combination $H_{n}, H_{n}^{*}$ is very similar to the well known configuration of a pair of regular tetrahedra symmetrical with respect to their common centroid. A more convenient but closely related geometric configuration is obtained as follows. Choose a geometric simplex $\bar{\sigma}_{n+1}$ in a euclidean space $S_{n+1}$, call $G$ its centroid, and $\bar{\sigma}, \cdots$ the images in $\bar{\sigma}_{n+1}$ of the abstract elements $\sigma, \cdots$. The linear spaces joining $G$ with the faces of $\bar{\sigma}_{n+1}$ decompose the boundary $\bar{H}_{n}$ into the elements of $\bar{H}_{n}{ }^{\prime}$. Let $P, P^{\prime}$ be the intersections with $\bar{H}_{n}$ of any line through $G$. Through the radial transformation $\bar{\rho}: P \rightarrow P^{\prime}$, the simplexes of $\left(\bar{\sigma}_{p}^{-1} \cdot \bar{\sigma}_{n+1}\right)^{\prime}$ go into those of $\left(\bar{\sigma}_{p}\right)^{*}$. The latter is therefore an $(n-p)$-cell transverse to $\bar{\sigma}_{p}$ and intersecting it in its centroid. These facts follow at once from the expressions of the simplexes of $\bar{\sigma}_{p}^{-1 \prime}$ and $\left(\bar{\sigma}_{p}\right)^{*}$. We observe also that the spaces $\bar{\sigma}, \bar{\sigma}^{*}$ are actually orthogonal, so that here both duality and "algebraic" orthogonality correspond to geometric orthogonality.

The geometric transformation $\bar{\rho}$ determines a simplicial transformation $\rho: H_{n}^{\prime} \rightarrow\left(H_{n}^{-1}\right)^{\prime}$, hence also $H_{n}^{*} \rightarrow H_{n}^{-1}$, characterized by permuting the vertices of $H_{n}^{\prime}$ associated with $\sigma_{p}$ and $\sigma_{p}^{-1} \sigma_{n+1}$.

5. Chain-Products. Generally speaking we shall understand by chain-product a chain-function $C \odot D$ of two chains $C, D$ with the following properties:

Postulate I. $C \odot D$ is a bilinear function of $C$ and $D$;

Postulate II. $\operatorname{dim}(C \odot D)=\operatorname{dim} C+\operatorname{dim} D$;

Postulate III. $F(C \odot D)=F(C) \odot D+(-1)^{\operatorname{dim} C} C \odot F(D)$.

The following noteworthy instances justify the definition. $\ddagger$

$\dagger \mathrm{I}$ am indebted for this remark and also for suggesting the radial transformation $\bar{\rho}$ below to one of my students, Mr. Shaun Wylie.

$\ddagger$ The three types (a), (b), (c) of Topology were unified by Tucker into a single w-multiplication, differing only from the above in that the power of $(-1)$ was $w+\operatorname{dim} C$, with a different $w$ in each case. 
(a) Intersections of chains. Let $C^{*}$ denote the result of replacing in $C$ every $\sigma$ by its dual $\sigma^{*}$. We have developed (loc. cit.) a complete combinatorial theory for the intersections of chains $D$ and $C^{*}$, written $D \cdot C^{*}$. Then our postulates are fulfilled with $C^{*} \odot D=D \cdot C^{*}$.

(b) Euclidean-products. This operation on two complexes is the abstract equivalent of the topological or euclidean product of their geometric realizations and plays an essential role in problems of fixed points (Topology, Chapter VI).

(c) Joins of two simplicial complexes. Let $K=\{\sigma\}, K^{\prime}=\left\{\sigma^{\prime}\right\}$ be two augmented complexes. Their join $K K^{\prime}$ is the augmented complex $\left\{\sigma \sigma^{\prime}\right\}$. If $C, D$ are chains of $K, K^{\prime}, C D$ is a definite chain of $K K^{\prime}$, which as a function of both satisfies our three postulates.

(d) The products of Cech and Whitney. $\dagger$ They are reducible to intersections combined with certain piece-wise simplicial transformations. We are back to $H_{n}$, and adopt a fixed order for its vertices. We agree also that in any expression $a_{i} a_{j} \cdots a_{k}, \sigma$, $(i, j, \cdots, k), \cdots$, the indices are always to be taken in ascending order.

Now there is a simplicial transformation $\tau: H_{n}^{\prime} \rightarrow H_{n}$ defined by $(i, j, \cdots, k) \rightarrow a_{k}$. When the vertices in $\sigma a$ are consecutive beginning with $a_{0}$ we readily verify

$$
\tau(\sigma a)^{*}=a F\left((\sigma a)^{-1} \sigma_{n+1}\right) .
$$

Consider now a product $\sigma a \sigma^{\prime}$, where the vertices are also consecutive beginning with $a_{0}$ and let $\odot$ denote the intersection operation of (a). We verify:

$$
\begin{aligned}
\tau\left((\sigma a)^{*} \odot \sigma a \sigma^{\prime}\right) & =a \sigma^{\prime}, \\
\tau\left[\left(a \sigma^{\prime}\right)^{*} \odot \tau(\sigma a)^{*}\right] & =\tau\left(\sigma a \sigma^{\prime}\right)^{*} .
\end{aligned}
$$

$\dagger$ The earlier product of Alexander-Kolmogoroff must be mentioned here. See notably Alexander, Annals of Mathematics, (2), vol. 37 (1936), pp. 698708; Cech, ibid., pp. 681-697. The connection of these recent products with my intersection theory has just been carried out by Wylie by a scheme analogous to the one utilized below. It explains why they are so numerous and how they are interrelated. The Alexander-Kolmogoroff ring based on these products is identical with the ring obtained by Gordon (Annals of Mathematics, (2), vol. 37 (1936), pp. 519-525) on the basis of our topological intersection theory as proved by Freudenthal in a forthcoming paper in the Annals of Mathematics. Since writing the above, I have found that the methods and results of Freudenthal and Wylie are substantially similar. 
If the indices of $\sigma a \sigma^{\prime}$ are not consecutive but merely in increasing order, we again obtain (9) and (10) provided that $\tau$ is replaced by the analogous operation obtained when the order of the indices is: first those of $\sigma a \sigma^{\prime}$, then the rest, in increasing order in each case. Finally in all other cases but those considered the operations performed at the left in (9) and (10) give zero for result.

We introduce now a new operation $\otimes$ defined in the following cases

$$
\begin{aligned}
(\sigma a)^{-1} \otimes \sigma a \sigma^{\prime} & =a \sigma^{\prime}, \\
(\sigma a)^{-1} \otimes\left(a \sigma^{\prime}\right)^{-1} & =\left(\sigma a \sigma^{\prime}\right)^{-1},
\end{aligned}
$$

whenever the factors in $\sigma a \sigma^{\prime}$ are in increasing order, while otherwise

$$
\sigma^{-1} \otimes \sigma^{\prime}=\sigma^{-1} \otimes \sigma^{\prime-1}=0 .
$$

The products $C^{\prime}=C^{-1} \otimes D$, and $C^{\prime \prime-1}=C^{-1} \otimes D^{-1}$ are defined for all $C$ and $D$ by imposing Postulate I. The other two are readily verified.

The coefficients of the chains which are multiplied must be such that the operations indicated have meaning: namely members of a unique ring, or two additive groups between which multiplication is defined, and so forth.

Kronecker-Index. The intersection $\sigma \cdot \sigma^{*}$ is a single vertex of $H_{n}^{\prime}$ taken \pm 1 times if $\sigma^{*}$ is the dual of $\sigma,=0$ otherwise. The exact coefficient, $\pm 1,0$, is called the Kronecker-index of $\sigma$ and $\sigma^{*}$, and designated by $\left(\sigma \cdot \sigma^{*}\right)$. From there we derive $\left(C_{p} \cdot C_{p}^{*}\right)$ by the additive property. The actual intersection is a zero-chain, and the index is the sum of its coefficients.

If we designate by $\left(C C^{\prime}\right)$ the constant term in the formal (algebraic) product of $C$ and $C^{\prime}$, then

$$
\begin{aligned}
\left(\sigma \cdot\left(\sigma^{\prime}\right)^{*}\right) & =\left(\sigma \sigma^{\prime-1}\right), \\
\left(C_{p} \cdot C_{p}^{\prime *}\right) & =\left(C_{p} C_{p}^{\prime-1}\right) .
\end{aligned}
$$

Therefore the non-vanishing of the formal product (15) is a sufficient condition that $C, C^{\prime}$ have common $p$-simplexes.

6. Application to Simplicial Complexes. We return to our simplicial complex $K$ with its vertices $a_{0}, \cdots, a_{r}$. We increase them by $a_{r+1}, \cdots, a_{n+1}, n$ arbitrary, and construct $H_{n}$ as before. The 
cells $\sigma^{*}, \sigma \in K$, constitute this time a sub-complex of $H_{n}^{*}$, and it is this complex which we denote by $K^{*}$. As for the abstract dual of $K$, we designate it by $K^{-1}$. We find by direct verification that if we preserve everywhere merely the cells of $K, K^{*}$, and apply our operations only to these, then all the results obtained so far continue to hold. Thus in (9) we must have $\sigma a \sigma^{\prime} \in K$, and in (10), we write zero at the right whenever $\sigma a \sigma^{\prime}$ does not belong to $K$. The different abstract products of $\$ 5$ will be defined as before with $F$ as the proper operator for $K$ and $K^{*}$, and for all multiplications to which it has been applied. We deduce directly from $\$ 5$, Postulate III, that all chain-products have the following common properties:

A. The product of two cycles is a cycle;

B. If one of the factor cycles in a product of two cycles is a boundary-cycle so is the product.

Property B implies also that the homology class of the product of two cycles is uniquely determined by those of the factors. This is the basic property in the proof of the topological invariance or of the invariance under subdivision of intersectioncycles. It may of course be utilized for the same purpose for the other multiplications.

Manifolds. Manifolds may be fitted readily in to our scheme. Roughly speaking if we wish to characterize $K$ as a $p$-manifold, we merely need to require that if $\sigma \in K$, and $S t(\sigma)$ is its star, then $S t(\sigma) \cdot \sigma^{*}=\zeta$ is a $p$-circuit $\bmod K-S t(\sigma)$ (Topology, Chapter III). It will follow that the intersections $\zeta \cdot \sigma_{q}^{*}$ determine $(p-q)$-chains which are related to the $\sigma_{q}$ 's like the duals. On the other hand they may also serve to determine the Betti groups and the like, and there follow the usual duality theorems for manifolds (Topology, Chapter III).

Geometric Realization. The notations being as in $\$ 4$, we find by means of the symbolic expressions of the cells, that the simplexes of $\bar{K}^{*}$ make up the $\bar{H}_{n}^{\prime}$-neighborhood of $\bar{K}$. If $\Phi$ is the boundary of the neighborhood then the cycles of $\bar{K}^{*}$ are those of this neighborhood mod $\Phi$. Their homology relations and the like are those of $\bar{K} \bmod \bar{H}_{n}-\bar{K}$. This confirms our assertion that the dual complex is tied up with those immersion properties of $\bar{K}$ in any $n$-sphere which are independent of $n$. The topological invariants of $\bar{K}^{*}$ (Betti groups and their invariants) are thus found to be topological properties of the complementary 
domains of $\bar{H}_{n}-\bar{K}$, depending solely upon the topology of $\bar{K}$, but not upon $n$. The simplest of these invariant properties is Alexander's duality theorem for the Betti numbers of $\bar{K}$ and of $\bar{H}_{n}-\bar{K}$. If we introduce orthogonal groups of coefficients we have the corresponding extensions for the Betti groups due to Pontrjagin.

\section{Correspondences and Transformations}

7. Product Complexes. Let $A, B$ be two complexes and $T, U$ two transformations $A \rightarrow B$. What elements if any are transformed alike by $T$ and $U$ ? This is essentially the problem of the coincidences. We have given coincidence and fixed point formulas for geometric complexes and certain abstract spaces (Topology Chapters VI, VII), and Tucker (loc. cit.) has completely treated the abstract complex. We shall consider the simplicial case only, where, owing to the negative dimensions, the treatment may be made particularly simple.

Let then $A, B$ be two finite closed simplicial complexes and let $a_{i}, b_{j}$ be their vertices. We augment both $A$ and $B$ so that the dimension of any simplex is equal to the number of its vertices.

Let $\mathfrak{A}$ and $\mathfrak{B}$ be the algebras of the type of $\S 3$ corresponding to $A, B$, and let $\alpha_{i}, \beta_{j}$ be the monomials of $\mathfrak{A}, \mathfrak{B}$ describing the simplexes of $A, B$. We shall denote by $\alpha^{i}$ the left-inverse of $\alpha_{i}$ and by $\beta^{i}$ the right-inverse of $\beta_{j}$, so that

$$
\alpha^{i} \alpha_{i}=\beta_{j} \beta^{i}=1, \quad \text { ( } i \text { not summed). }
$$

The elements $\alpha^{i}, \beta^{j}$ are those of the duals $A^{-1}, B^{-1}$.

We now introduce a new algebra $\mathfrak{A} \mathfrak{B}$, composed of $\mathfrak{A}, \mathfrak{B}$, whose base terms are $\alpha_{i} \beta^{i}, \alpha^{i} \beta_{j}, \pm 1,0$, the monomials indicated being zero unless each factor $\neq 0$. We verify under the circumstances that the aggregates

$$
A B^{-1}=\left\{\alpha_{i} \beta^{j}\right\}, \quad A^{-1} B=\left\{\alpha^{i} \beta_{j}\right\}
$$

treated as if all the factors were simplexes, are dual to one ananother with $\alpha_{i} \beta^{i}, \alpha^{i} \beta_{j}$ as corresponding dual elements. $\dagger$

$\dagger$ Our procedure may be made more "geometric" by completing $A, B$ to spheres $H_{n}{ }^{a}, H_{n}{ }^{b}$, with $\sigma_{n}{ }^{a}+1, \sigma_{n}{ }^{b}+1$ as their simplexes, then taking the simplexes $\left\{\alpha_{i} \beta_{i}^{-1} \sigma_{n}^{b}{ }^{b}\right\},\left\{\sigma_{n+1}^{a} \alpha_{i}^{-1} \beta_{j}\right\}$ of the join $H^{a} H^{b}$. It may be observed that the chief advantage of our treatment of these questions over Tucker's is that practically no computations are necessary to show that various cell-aggregates encountered are complexes. 
As a rule when we have a combination such as $\alpha_{i} \beta^{j}$, it shall be understood that $\operatorname{dim} \alpha_{i}=-\operatorname{dim} \beta^{j}$, and $\operatorname{dim} \alpha_{i}$ shall be denoted by $p$.

8. Correspondences. By a correspondence $\mathcal{S}_{a}{ }^{b}$ between $A$ and $B^{-1}$ over a coefficient-ring $(\$)$, is meant an aggregate of triples

$$
\mathfrak{C}_{a}{ }^{b}: \quad\left\{\alpha_{i}, \beta^{j}, x_{j}{ }^{i}\right\}, \quad x_{j}^{i} \in \text { SS. }
$$

The numbers $x$ may be thought of as weights with which the couples are taken.

$\mathbb{S}_{a}^{b}$ determines the chain of $A B^{-1}$ :

$$
C_{a}^{b}=x_{j}^{i} \alpha_{i} \beta^{j},
$$

and also a dual correspondence between $A^{-1}$ and $B$ :

$$
\mathfrak{S}_{b}{ }^{a}: \quad\left\{\alpha^{i}, \beta_{j}, \bar{x}_{i}^{j}\right\}, \quad \bar{x}_{i}^{j}=x_{j}{ }^{i},
$$

whose representative chain in $A^{-1} B$ :

$$
C_{b}^{a}=x_{i}^{j} \alpha^{i} \beta_{j},
$$

is dual to (18) except for possible change in sign. Notice that if $x_{p}, \bar{x}_{p}$ denote the matrices of the coefficients $x, \bar{x}$ for $\operatorname{dim} \alpha_{i}=p$, then $\bar{x}_{p}=x_{p}^{\prime}$.

Let $\mathfrak{D}_{a}^{b}$ be a second correspondence with $D, y$ in place of $C, x$. We inquire whether $\mathfrak{C}_{a}{ }^{b}$ and $\mathfrak{D}_{a}{ }^{b}$ possess coincident couples, that is, whether some $\left(\alpha_{i}, \beta^{j}\right) \in \mathfrak{S}_{a}^{b}$ and also $\in \mathfrak{D}_{a}^{b}$. We shall then say that there is a coincidence of $\mathfrak{S}_{a}^{b}$ and $\mathfrak{D}_{a}^{b}$. A necessary and sufficient condition for such an occurrence is that

$$
(-1)^{p} x_{j}{ }^{i} \bar{y}_{i}^{j}=x_{j}^{i} \alpha_{i} \beta^{j} \cdot \bar{y}_{i}^{j} \alpha^{i} \beta_{j} \neq 0, \quad(i, j \text { unsummed }) .
$$

A sufficient condition for the occurrence of some coincidence is, from (5), that

$$
-\Theta=\left(C_{a}^{b} D_{b}^{a}\right) \neq 0,
$$

where $(C D)$ represents the numerical term in this product, or the Kronecker-index of the chains. $\Theta$ is the algebraic number of coincidences. By actual computation

$$
-\Theta=\sum(-1)^{p} x_{i}{ }^{j} \bar{y}_{j}{ }^{i}=\sum(-1)^{p} \text { trace } x_{p} y_{p}^{\prime} .
$$

We might obviously assume that the coefficients $x, y$ belong respectively to groups $\left(\mathcal{H}, \mathcal{B}^{\prime}\right.$ ' between which a distributive multiplication is defined (for example, $\mathcal{H}^{*}, \mathcal{S}^{\prime}$ are character groups of one another). 
It is clear that the present result could only be of use in topology whenever $\Theta$ is expressible in topologically invariant terms. Since the only known chains with topological character are cycles, we are led to the following very natural assumption.

Assumption I. The representative chains $C_{a}{ }^{b}, D_{b}{ }^{a}$ are both cycles (Topology, Chapter VI).

It is also expedient to add another assumption.

Assumption II. The ring $\$$ is the field of all rational numbers.

Now the bases $\alpha, \beta$ for the chains of $A$ and $B^{-1}$ have been chosen "orthogonal" throughout, in the sense that

$$
\alpha^{i} \alpha_{j}=\beta_{i} \beta^{j}=\delta_{i}{ }^{j} \quad(\text { the Kronecker } \delta) .
$$

If we impose contragredient transformations upon the bases our results will be left formally. unchanged.

Under the circumstances, if we introduce suitable canonical bases with the non-bounding cycles duly isolated, the only terms left in the expressions of the chains $C, D$ will be products of such cycles. Returning now to the non-augmented complexes, with dimensions counted in the usual way, if $X_{i}{ }^{j}, Y_{j}{ }^{i}$, denote the coefficients in the transformation of the independent cycles, and their matrices are denoted by $X_{p}, Y_{p}$, we shall have

$$
\Theta=\sum(-1)^{p} \text { trace } X_{p} Y_{p}^{\prime} \text {. }
$$

9. Transformations. A transformation $T_{a b}: A \rightarrow B$ over (S) is a collection of homomorphisms between chain-groups determined by relations

$$
T_{a b}: \quad \alpha_{i} \rightarrow x_{i}{ }^{j} \beta_{j} ; \quad x \in \text { (S); } \operatorname{dim} \alpha_{i}=\operatorname{dim} \beta_{j} .
$$

It determines a dual transformation $T^{a b}$ and two dual correspondences $\mathfrak{C}_{a}{ }^{b}, \mathcal{C}_{b}{ }^{a}$ :

$$
T^{a b}: \quad \alpha^{i}=\bar{x}_{j}^{i} \beta^{j}, \quad \bar{x}_{j}^{i}=x_{i}^{j} ;
$$

$$
\mathfrak{S}_{a}^{b}: \quad\left(\alpha_{i}, \beta^{j} ; \bar{x}_{j}^{i}\right), \quad \mathfrak{S}_{b}^{a}: \quad\left(\alpha^{i}, \beta_{j} ; x_{i}^{j}\right) .
$$

Let $U_{a b}$ be a second $T$ with $\mathfrak{D}, y$ in place of $\mathfrak{C}, x$. A coincidence of $T_{a b}$ and $U_{a b}$ is a couple $\left(\alpha_{i}, \beta_{j}\right)$ such that $\beta_{j} \in T_{a b} \alpha_{i}$ and also $\epsilon U_{a b} \alpha_{i}$. They correspond to the coincidences of $\mathfrak{E}_{a}^{b}$ and $\mathfrak{D}_{a}^{b}$. 
Hence $\Theta \neq 0$ is also a sufficient condition for the existence of some coincidence of $T_{a b}$ and $U_{a b}$. The matrices $x_{p}, y_{p}$ are now the transformation matrices for the $p$-cells, and $X_{p}, Y_{p}$, those for the $p$-cycles. Here also restrictions are necessary. Now it follows from Assumption I that if $\alpha, \beta$ are cycles, then $C_{a}{ }^{b} \beta$ and $\alpha D_{b}{ }^{a}$ are both cycles. In order that this hold, we find that the following assumption is a necessary and sufficient condition.

Assumption $\mathrm{I}^{\prime} . T_{a b}$ and $U^{a b}$ are permutable with the boundary operator $F$.

This is Tucker's equivalent of our earlier Assumption I.

Suppose now that $T, U$ are transformations of $A$ into itself. We introduce another copy $B$ of $A$, replace $T, U$ by transformations $A \rightarrow B$, and then apply our formulas. In particular, if $U=1$, we have $y_{p}=1, Y_{p}=1$, and hence

$$
\Theta=-\sum(-1)^{p} \text { trace } x_{p}=\sum(-1)^{p} \text { trace } X^{p} .
$$

The equality between the sums in (22), (23) is a so-called generalized Euler-Poincaré relation in the sense of Hopf and Tucker. When $T=U=1$ it reduces in fact to the ordinary Euler-Poincaré relation.

10. Coincidences and Fixed Points of Transformations of Geometric Complexes. In formulas (23) and (24) one may readily recognize the abstract equivalent of (23), (28), (29) of Topology, Chapter VI, our early coincidence and fixed point formulas for geometric manifolds and complexes. Let us show how the present algebraic procedure is related to our former more topological method.

Let $A$ be a closed simplicial complex, and $A_{0}$ a topological image of $A$ in a space $S_{n}$. If $\left\{\gamma_{p}^{i}\right\}$ is a base for its rational $p$-cycles and $C_{n-p}$ a cycle of $S_{n} \bmod S_{n}-A_{0}$, the relations

$$
\left(\gamma_{p}^{i} \cdot C_{n-p}\right)=x_{i}, \quad\left(x_{i} \text { rational }\right),
$$

uniquely determine a homology class for $C_{n-p}$. The aggregate of all these classes for all the possible choices of $A_{0}, S_{n}$, is the rational $(-p)$-dual cycle $\gamma^{p}$ determined by the numbers $x_{i}$. We write $\gamma^{p} \sim 0$ when every $x_{i}=0$ and set $\left(\gamma_{p}{ }^{i} \cdot \gamma^{p}\right)=x_{i}$. These dual cycles (called pseudocycles in our earlier work), form an additive abelian group. Dual cycles and groups are thus introduced 
directly without passing through the medium of the chains, by means of their "orthogonality" to the absolute cycles.

Suppose now that we have two geometric complexes $A, B$ undergoing two transformations $T, U$ into one another. By a coincidence of the two is meant a couple of points $x \in A, y \in B$, such that $y \in T x, y \in U x$. Does there exist an invariant analogous to $\Theta$ such that when it is $\neq 0$ there is at least one coincidence?

Let us identify $A, B$ or subdivisions of them with geometric complexes which are subcomplexes of two euclidean regions $\Omega_{n}{ }^{a}$, $\Omega_{n}{ }^{b}$ subdivided into simplexes of diameter $<\epsilon$ assigned. Let $N_{a}, N_{b}$ be the $\Omega^{a}, \Omega^{b}$ neighborhoods of $A, B$. Let us designate by $\alpha_{i}, \beta_{i}$ and $\alpha^{i}, \beta^{i}$ the $p$-cycles and $(-p)$-dual cycles of $A, B$. We assume them represented by obvious simplicial cycles.

Let $S_{2 n}$ be the product of the spaces of $A$ and $B$. The chains $\alpha^{i} \times \beta_{j}$ and $\alpha_{i} \times \beta^{j}$ characterize two dual types of cycles of $S_{2 n}-A \times B$, which we shall call $\Gamma, \Delta$. Let us suppose that $T, U$ may be extended to transformations $\bar{T}, \bar{U}: N_{a} \rightarrow N_{b}$, having the following properties:

(a) the transformations $A \rightarrow B$ which they determine are $T, U$; (b) the coincidences of $\bar{T}, \bar{U}$ are the same as those of $T, U$; (c) the sets in $S_{2 n}$ which describe $\bar{T}, \bar{U}$ coincide with two singular relative cycles $\Gamma, \Delta$, of $S_{2 n} \bmod S_{2 n}-A \times B$, respectively expressible (in the sense of homology) in terms of the cycles of types $\alpha_{i} \times \beta^{j}$ and $\alpha^{i} \times \beta_{j}$. Under the circumstances the Kroneckerindex $(\Gamma \cdot \Delta)$ is a suitable invariant $\Theta$. For $(\Gamma \cdot \Delta) \neq 0$ implies that $\Gamma, \Delta$ intersect, hence that $\bar{T}, \bar{U}$ have a coincidence and by (b) this must hold regarding $T, U$. In fact if we express $\Gamma, \Delta$ respectively in terms of the cycles, $\alpha^{i} \times \beta_{j}$ and $\alpha_{i} \times \beta^{j}$, and apply my intersection theory (Topology, Chapter IV), we obtain precisely (23).

The simplest case is where $T$ is a continuous single-valued transformation (=c.s.v.t.). We then choose $N_{a}, N_{b}$ normal (Topology, p. 91). That is to say, for example, through every point $P$ of $N_{a}-A$ there passes a unique projecting segment with endpoints $Q$ on $A$ and $R$ on the boundary of $N_{a}$. We then choose $\bar{T}$ such that $\bar{T} \cdot Q R=T \cdot Q$. It turns out that $\bar{T}$ has for image in $S_{2 n}$ a $\Gamma$. We choose for $U$ a transformation such that $U^{-1}$ is of the type of a c.s.v.t. and extend it to a $\bar{U}$ in a similar manner with the roles of $A, B$ interchanged. The image of $\bar{U}$ is then a $\Delta$.

Special case. $B$ is a copy of $A, U$ is $A \rightarrow B$ corresponding to the 
identical transformation $A \rightarrow A$. Then $\bar{U}$ may be chosen of type $\Delta$, and the number $(\Gamma \cdot \Delta)$ obtained is precisely (24).

Let us recall in concluding that the same formulas hold for transformations of compact metric HLC spaces. They are spaces endowed with a strong type of local connectedness in the sense of homology, analogous to that possessed by the so-called absolute neighborhood retracts. $\dagger$

Princeton University

\section{CIRCLES IN WHICH $|F(x)|$ HAS A SINGULARITY OR ASSUMES PREASSIGNED VALUES}

BY J. W. CELL

Let $k$ be a given positive integer and let $a_{0}$ and $a_{k} \neq 0$ be two given constants. Let $F_{k}(x)$ be any member whatever of the class $C_{k}$ of functions which are regular in the neighborhood of the origin and which there have the expansion

$$
F_{k}(x)=a_{0}+a_{k} x^{k}+a_{k+1} x^{k+1}+\cdots,
$$

where $a_{0}$ and $a_{k}$ are the two given constants.

Theorem 1. Let $\eta\left(a_{0}, a_{1}\right)=0$ if $\left|a_{0}\right|=1$. In case $\left|a_{0}\right|<1$, let $\eta\left(a_{0}, a_{1}\right)=\left\{1-\left|a_{0}\right|^{2}\right\} /\left|a_{1}\right|$, and if $\left|a_{0}\right|>1$, let $\eta\left(a_{0}, a_{1}\right)=$ $\left\{2\left|a_{0}\right| \log \left|a_{0}\right|\right\} /\left|a_{1}\right|$. Then in or on the circle $|x|=\eta\left(a_{0}, a_{1}\right)$, either $F_{1}(x)$ has a singularity or $\left|F_{1}(x)\right|$ assumes the value one. Moreover, no smaller radius will do for the whole class of functions $C_{1}$.

Corollary. $\eta\left(a_{0}, 1\right)=\left|a_{1}\right| \eta\left(a_{0}, a_{1}\right)$.

Proof. If $\left|a_{0}\right|=1$, the theorem is granted, so we shall henceforth suppose that this is not the case. If $a_{0}=r e^{i \alpha},(r \geqq 0)$, we define $E(x)=e^{-i \alpha} F_{1}(x)$. Then $|E(x)|=\left|F_{1}(x)\right|$ and hence we may, with no loss of generality in the proof, suppose that $a_{0}$ is real and non-negative.

CASE $1.0 \leqq a_{0}<1$. There exists a positive number $\eta$ such that for $|x| \leqq \eta, F_{1}(x)$ is regular and $\left|F_{1}(x)\right|<1$. Now form

$$
G(x)=\frac{F_{1}(x)-a_{0}}{-a_{0} F_{1}(x)+1} .
$$

† See Duke Mathematical Journal, vol. 2 (1936), pp. 435-442. 